\title{
Implementation and Study of Sound Control LED Switch
}

\author{
Wen-Bin Lin ${ }^{1}$, Kao-Feng Yarn ${ }^{2}$ \\ ${ }^{1}$ Department of Electronic and Optoelectronic Application Engineering, ${ }^{2}$ Department of Aircraft Maintenance, \\ Far East University, Taiwan 744, ROC \\ Corresponding Author: Wen-Bin Lin
}

\begin{abstract}
In this paper, the sound control application is taken as the research content of this implementation. Finally, the operating principle of the adopted electric circuits and the performance of the proposed device are validated by experimental results. It reveals that the operations of the proposed device coincide with the requirements.
\end{abstract}

Keywords: Sound control switch, LED, MIC, Verification

\section{INTRODUCTION}

Nowadays, social science and technology are changing with each passing day, and electronic products are already an inevitable trend. All kinds of electronic products and new inventions are nothing more than to make people's lives more convenient and comfortable.

Before going to bed every night, we always lie down in bed for a while, and then wake up and turn off the lights to go to bed when we feel sleepy. But we always feel that it's a little troublesome to get up and turn off the lights, especially in cold weather, and we don't want to leave the bedside. So we wanted to find a way to turn off the lights without getting up-a remote control circuit. After literatures reviewing, we found that there are roughly two methods [1-6]: One is to use infrared remote control, the other is to use sound control switches. Infrared remote control is just like using a home appliance, using the remote control to control it. The sound control switch, as the name implies, uses sound to achieve the purpose of controlling the switch. We compare the two methods; the sound control switch is more in line with our needs. Because it does not require additional power except for the receiving end.

If we use infrared remote control, once the remote control is not around, we have to leave the bedside, which loses the meaning of the original circuit requirements. So we chose to make and research sound control switches. We hope to use the characteristics of LED and MIC to play the function of sound control switch.

The sound control part uses a condenser microphone (Condenser Mic). There is a field effect transistor inside the condenser microphone; the purpose is to change the output impedance. Because of its small size, many electronic products are used. The basic principle is to use the principle that sound is air vibration to generate electronic signals. For example, radio microphones or other piezoelectric components. Then use these electronic signals generated by the vibration to control the switch.

\section{BASIC PRINCIPLES AND DESIGN METHODS}

As shown in the hardware circuit diagram in Figure 1, its functions are described as follows: The basic principle uses a class A amplifier as the pre-stage sound amplifier circuit. First of all, M300 is the capacitor is MIC. The biggest advantage 
of capacitive MIC is high sensitivity. Like the transistor, because the capacitive MIC must provide a bias voltage (the dynamic microphone is not necessary). So it connects Vin with an R10. The sound vibrates the MIC, the tiny voltage that the MIC changes is coupled by $\mathrm{C} 1$ and is amplified by Q1 as the first stage. R2 and R3 form a self-bias mode (if R2 is connected to the Vin terminal, it is called a fixed bias). R6 is the current limiting resistor of Q1. VR1 is the load resistor of Q1. The first-level signal is adjusted by VR1, and is coupled to Q2 from $\mathrm{C} 2$ for second-level amplification, and the output of C3 is connected to a symmetrical drive circuit composed of Q3 and Q4. R8 and $\mathrm{C} 4$ are used as RC filters, mainly to prevent the LED from flickering; the ripple current will cause feedback and be amplified by the pre-stage, which has the effect of noise suppression. A symmetrical drive circuit composed of Q3 and Q4, Q3 provides drive current. When the audio signal sent by Q2 causes Q3 to produce an audio current change, Q4 generates a variable bias voltage through R5, causing the LED to produce a change in audio frequency.

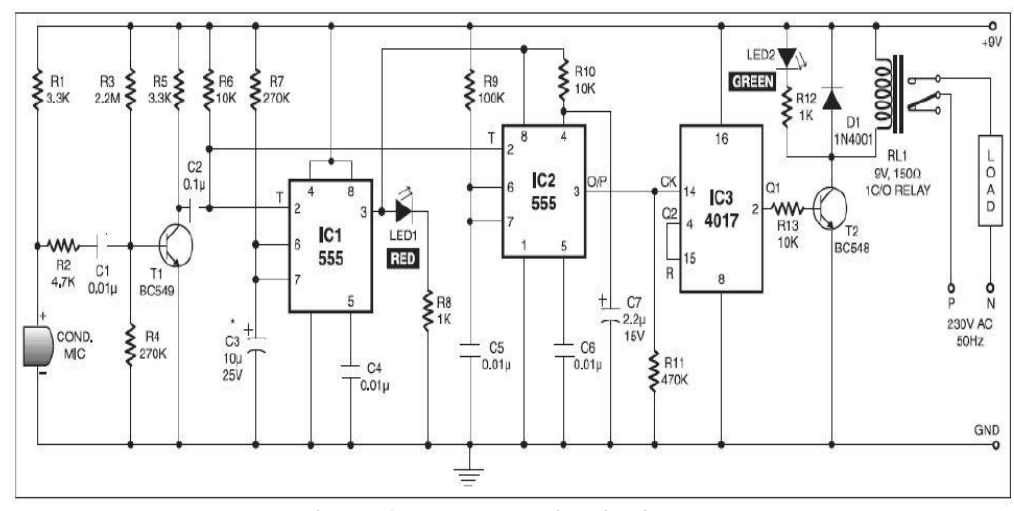

Figure 1: Hardware circuit diagram

The circuit in Figure 1 is composed of 555IC plus 4017IC. It takes two clapping sounds to turn the relay on and off, so it can reduce the occurrence of mis-operation. LED1 shows whether it is a preparatory operation, and LED2 shows whether the relay is on or not.

After trial-making with a breadboard, it was found that the circuit did not work properly. After analysis and discussion, the following modifications are made in Figure 1: As shown in Figure 2, the current limiting resistor $\mathrm{R} 1$ is changed to $10 \mathrm{k} \Omega$. Add a $6.8 \mathrm{k} \Omega$ resistor to the second pin of 555IC1 to ground. Change C5 to $0.1 \mu F, \mathrm{R} 10$ to $1 \mathrm{k} \Omega$, and the second pin of 555IC3 should be grounded.

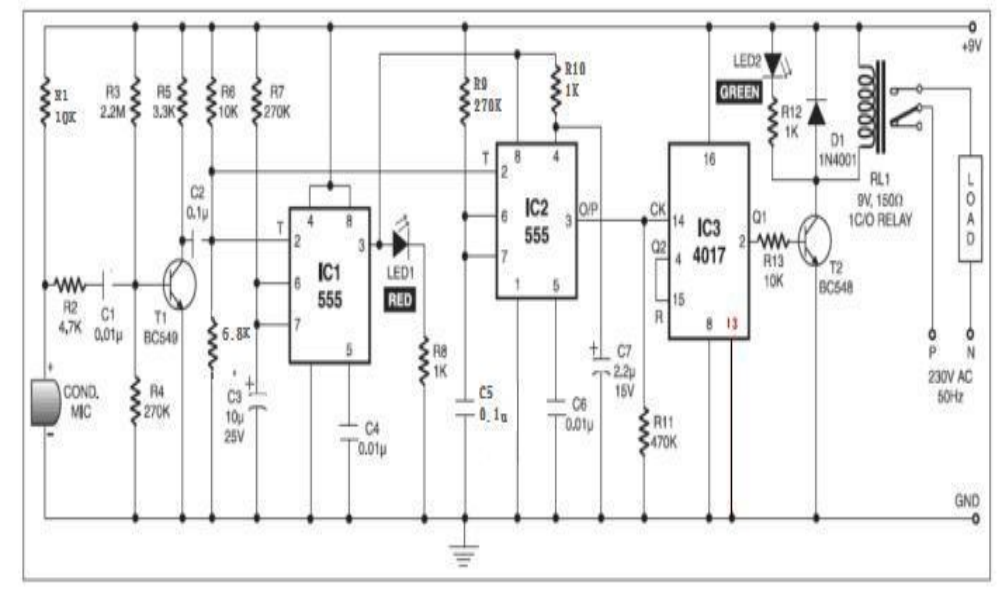

Figure 2: Modified hardware circuit diagram 
But in terms of system sensitivity, it still needs to be strengthened. We found that after the microphone input in Figure 1 circuit, it was only amplified by one stage, and it was unable to generate enough voltage to trigger the $555 \mathrm{IC} 1$ at a small volume. After analysis and discussion, if the microphone undergoes secondary amplification, the sensitivity of the circuit increases. After modification, the sensitivity of the circuit has increased a lot, but it is too sensitive. As shown in Figure 3, the solution is to reduce $\mathrm{C} 1, \mathrm{C} 2$, and $\mathrm{C} 3$. We changed them to $10 \mathrm{nF}$ capacitors. Figure 4 shows the breadboard of the voice control circuit after adding the secondary amplification.

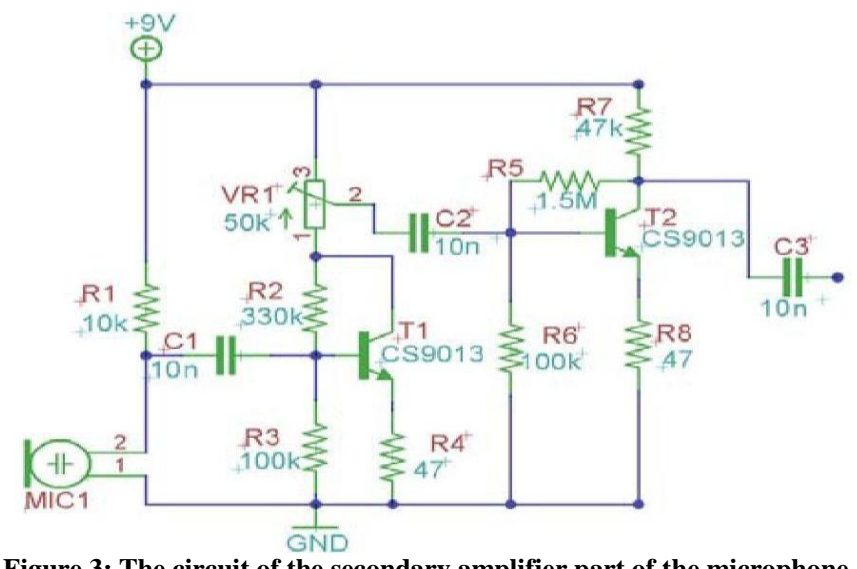

Figure 3: The circuit of the secondary amplifier part of the microphone

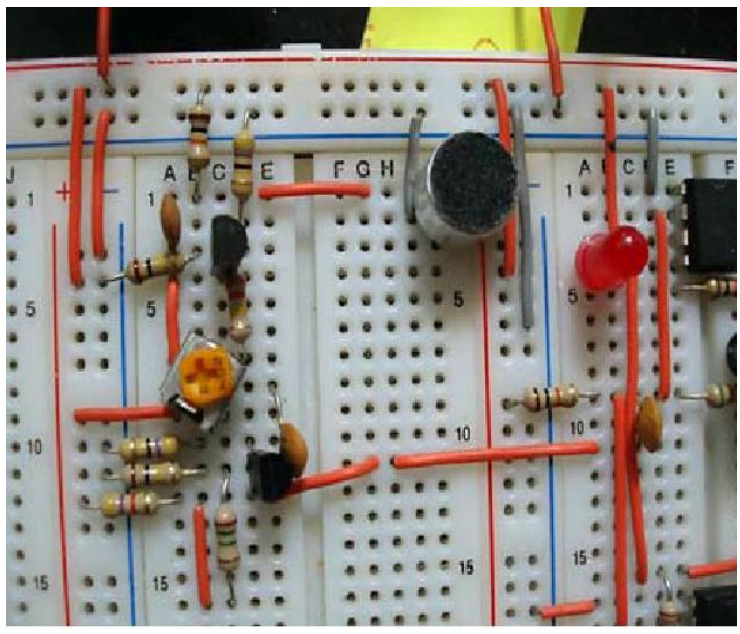

Figure 4: Add the second-level amplified voice control circuit breadboard

\section{CIRCUIT PRINCIPLE ANALYSIS \\ 3.1 Power Part}

The power supply adopts a capacitor step-down method. In AC, the capacitor will produce capacitive reactance. The larger the frequency or capacitance value, the smaller the capacitive reactance. The formula is as follows, $\quad X_{C}=\frac{1}{\omega C}=\frac{1}{2 \pi f C}$. Where, $X_{C}$ represents capacitive reactance, $\mathrm{C}$ represents capacitance, and $f$ represents frequency. The capacitance of this circuit is
$1.5 \mathrm{uF}$. If it is calculated at $110 \mathrm{~V}, 60 \mathrm{~Hz}$, then:

$$
\begin{aligned}
& \frac{1}{2 * \pi * 60 * 0000015 .} \\
& \cong \frac{1}{5.65486677646162782 \Phi 32758089931 \mathrm{e}^{-4}} \\
& =1768.388256576614841876486259646
\end{aligned}
$$

The calculated result is about 1768 ohms, which can be divided by the load resistance to get a voltage. After the Zener diode is regulated, $9 \mathrm{~V}$ power can be obtained. R17 750k ohm is used for $\mathrm{C} 1$ $1.5 \mathrm{uF}$ discharge. Because $\mathrm{V}=\mathrm{IR}$, the mathematical formula can be expressed as: $110=1768$ I. The maximum available current can be obtained $\cong 0.06221719$ (A), which is $62 \mathrm{~mA}$.

\subsection{Circuit}

\subsubsection{Microphone Signal Amplification}

As shown in Figure 5, R1 is the current limiting resistor of the microphone, and the signal is first-stage amplified by $\mathrm{T} 1$ after being coupled with $\mathrm{C} 1$. VR1, R2, and $\mathrm{R} 3$ form a self-sufficient bias voltage, and VR1 adjusts the microphone sensitivity. The signal is coupled to Q2 via C2 for second- 
stage amplification, and the signal is output from Q2 via C3 to the second pin of IC1 for

triggering.

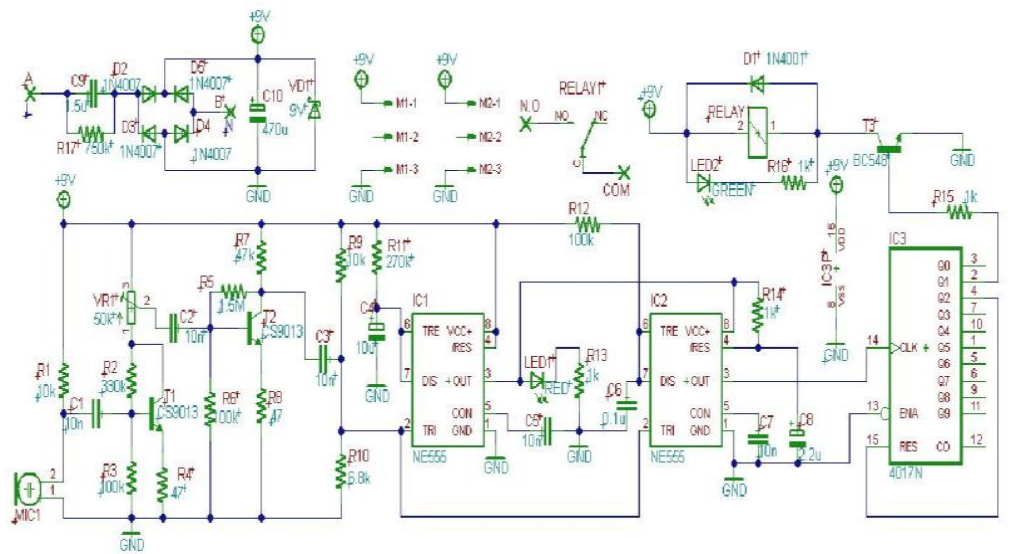

Figure 5: Sound control circuit with capacitive step-down and two-stage amplification

\subsubsection{First Applause}

Because the trigger pin (that is, the second pin) of the 555IC is lower than $\frac{1}{3} \mathrm{~V}_{\mathrm{CC}}$, the output pin of the third pin will be high and the seventh pin will be open. When the sixth pin is higher than $\frac{2}{3} \mathrm{~V}_{\mathrm{CC}}$, the output is low. When the signal triggers the second pin, the output of the third pin is high and LED1 is turned on. And provide IC2 power supply, ready to accept the second applause, do preparatory actions. At this time, C4 starts to charge. When the charge is greater than $\frac{2}{3} \mathrm{~V}_{\mathrm{CC}}$, the LED is turned off, and the IC2 power is turned off to cancel the preparatory action. The number of seconds of the preparation time is controlled by $\mathrm{C} 4$ and $\mathrm{R} 11$, which can be calculated as follows: Because the RC series transient formula is $\tau=\mathrm{RC}$, the values of $\mathrm{C} 4$ and $\mathrm{R} 11$ are taken in, and $\tau=270 \mathrm{k} * 10 \mathrm{u}=$ 2.7 (seconds). When $1 \tau$ (that is, 2.7 seconds have passed), the capacitor is charged to

$9\left(1-e^{-1}\right)=9\left(1-\frac{1}{2.71828}\right) \cong 9(1-0.368)=9 * 0.632$ $=5.688$ (voltage $)$. At this time it is close to, so the preparation time is about 2.7 seconds. If we want to shorten this time, just reduce the value of $\mathrm{C} 4$ or R11.

\subsubsection{Second Applause}

In order to prevent false triggering when IC1 provides IC2 power supply, R14 and $\mathrm{C} 8$ are added to the eighth and fourth pins of IC2. When the IC2 power is turned on, the third pin remains low state. When preparing for action, the second clapping clap triggers IC2, and makes the third pin high, triggering IC3. It makes the Q1 of IC3 high, and after the current limit by R15, makes the transistor operate, and LED2 will turn on. The relay operates to control the load side.

\section{PRODUCTION RESULTS}

As shown in Figure 6, the completed circuit connected to the breadboard. When the finished product is tested, the 555 IC1 lights up green when it is operating.

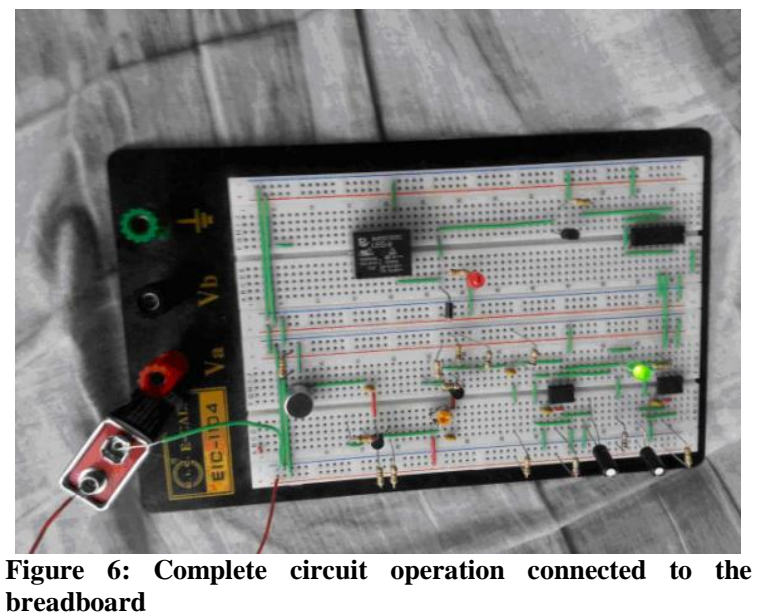


As shown in Figure 7, the completed circuit connected to the breadboard. When the finished product is tested, the red light on the 555 IC2 is active, which means that there is current conducting.

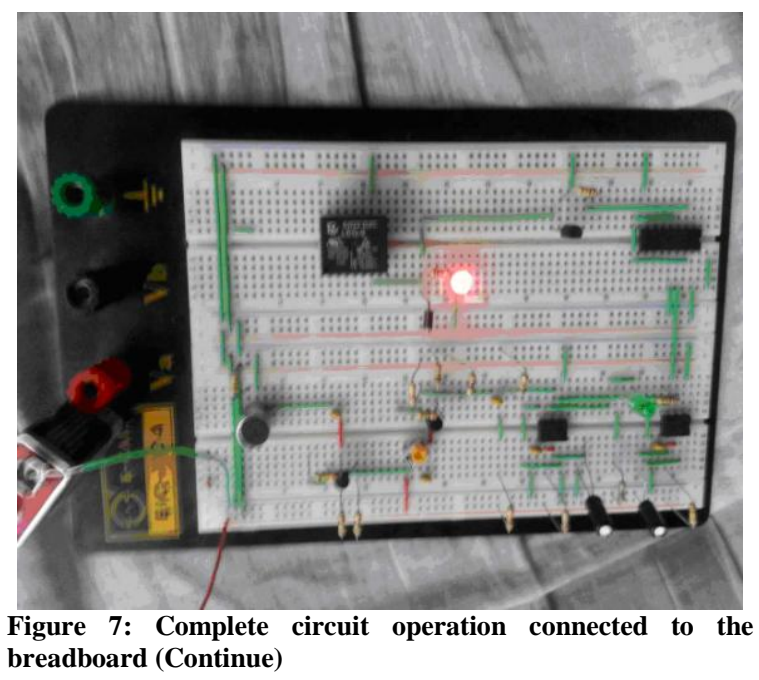

\section{CONCLUSION AND HARDWARE PRODUCTION RESULTS}

Figures 6 to 7 are photos of the hardware results of this paper. This paper implements a set of circuits that meet the above-mentioned functions, and uses the results of the implementation to verify the actions of the prototype circuit and the functions that meet the requirements.

The sound control circuit can conveniently control electrical products, without having to walk to the electrical appliances or use the remote control, just clap our hands. If the sound control circuit can be integrated into the light bulb, then it becomes a "sound control light bulb". Just use the sound to control the light bulb to turn on or off. But the problems to be overcome are: Assuming that we only want to turn off one of the bulbs, how to prevent the two bulbs from operating at the same time. As a result of our thinking, we thought that we could use the number of claps, or the time between two clapping, or even the way of clapping. For example, the tone of Do and Me turns on the first light bulb, and the tone of Me and So turns on the second light bulb.

\section{Acknowledgement: None}

\section{Conflict of Interest: None}

\section{Source of Funding: None}

\section{REFERENCES}

1. George Charles Electronic Circuit Network, The retrieval date is 2020/03/02. http://gc.digitw.com/new_page_5.htm.

2. George Charles Electronic Circuit Network, The retrieval date is 2020/03/02. http://gc.digitw.com/Program/VOLT-ONREG/VOLT-ON-REG.htm.

3. On-line calculation of resistance divider, The retrieval date is 2020/03/02. http://samengstrom.com/elec/resistor/index. html.

4. NE555 Timing IC, The retrieval date is 2020/03/02.

http://gc.digitw.com/Program/NE555-

CALC/555\%20Timer\%20Calculator.htm.

5. M.Z. Chen, Basic Electronics Practice (II), Zhixing Culture Publishing Co., Ltd., Taiwan, 2001.

6. W.Y. Li, Basic Electricity (II), Flag Information Co., Ltd., Taiwan, 2006.

How to cite this article: Wen-Bin Lin, Kao-Feng Yarn. Implementation and study of sound control LED switch. International Journal of Research and Review. 2021; 8(4): 191-195. DOI: https://doi.org/10.52403/ijrr.20210426 